\title{
Evaluating the Effect of Treatment Persistence on the Economic Burden of Moderate to Severe Psoriasis and/or Psoriatic Arthritis Patients in the U.S. Department of Defense Population
}

\author{
Seina Lee, MS, PharmD; Lin Xie, MS, MA; Yuexi Wang, ME; \\ Neel Vaidya, MS; and Onur Baser, MS, MA, PhD
}

\begin{abstract}
BACKGROUND: Psoriasis is a chronic, hyper-proliferative dermatological condition associated with joint symptoms known as psoriatic arthritis (PsA). In a 2013 review, the total economic burden of PsA was estimated at \$51.7$\$ 63.2$ billion. The economic burden of moderate to severe psoriasis patients has reduced significantly with the advent of biologics, but there remains a dearth of real-world evidence of the impact of treatment persistence on the economic burden of moderate to severe psoriasis and/or PsA patients.
\end{abstract}

OBJECTIVE: To evaluate the overall and psoriasis and/or PsA-related health care utilization and costs among patients who were persistent versus those nonpersistent on index biologic among the moderate to severe psoriasis and/or PsA population.

METHODS: Adult patients with $\geq 2$ claims with diagnosis of psoriasis and/or PsA during the period of November 2010-0ctober 2015 were identified from the U.S. Department of Defense database; the first diagnosis date during November 2011-0ctober 2014 was defined as the index date. As of the index date, patients were considered to have moderate to severe psoriasis or PsA if they had $\geq 1$ nontopical systemic therapy or phototherapy during the 1-year pre- or 1-month post-index date. Persistence to index therapy, defined as the first biologic used (etanercept, adalimumab, ustekinumab, infliximab) on or within 30 days post-index date, was determined based on the biologic dosing schedule and a 90 -day gap. Generalized linear models were used to compare the health care utilization and costs between persistent and nonpersistent patients during the 1-year post-index period.

RESULTS: A total of 2,945 moderate to severe psoriasis and/or PsA patients were identified. Of those, 1,899 (64.5\%) were persistent and 1,046 (35.5\%) were nonpersistent. Compared with nonpersistent patients, persistent patients were older ( 49.2 vs. 45.5 years; $P<0.001$ ) and more likely to be male (52\% vs. $45 \%$; $P<0.001$ ). More persistent patients were diagnosed with dyslipidemia ( $40 \%$ vs. $35 \%$; $P=0.002$ ), had lower antidepressant use ( $23.4 \%$ vs. $27.4 \% ; P<0.001$ ), and had lower anxiolytic use ( $30 \%$ vs. $37 \%$; $P<0.001)$ compared with nonpersistent patients. After adjusting for demographic and clinical characteristics, nonpersistent patients had higher total medical costs $(\$ 12,457$ vs. $\$ 8,964 ; P<0.001)$ compared with persistent patients, and ambulatory visits ( 23.9 vs. $21.4 ; P=0.007$ ) were a major contributor. Approximately $40 \%$ of the total overall medical costs were attributed to psoriasis and PsA. Although persistent patients incurred higher pharmacy costs $(\$ 10,684$ vs. $\$ 7,849 ; P<0.001)$ due to higher biologic use and the potentially high per-unit cost of biologics, their psoriasis- and/or PsA-related medical costs were significantly lower than those of nonpersistent patients $(\$ 3,395$ vs. $\$ 5,041 ; P<0.001)$. Total overall costs combining medical and pharmacy costs were similar between the cohorts $(\$ 22,678$ vs. $\$ 21,477 ; P=0.122)$.

CONCLUSIONS: Moderate to severe psoriasis and/or PsA patients who were persistent on index biologic treatment had higher pharmacy utilization and costs, albeit with lower medical costs and similar total costs, compared with nonpersistent patients.

\section{J Manag Care Spec Pharm. 2018;24(7):654-63}

Copyright $\odot 2018$, Academy of Managed Care Pharmacy. All rights reserved.

\section{What is already known about this subject}

Psoriasis has a significant clinical and economic burden among children, adolescents, and adults, with $\$ 51.7-\$ 63.2$ billion in total direct costs attributed to psoriasis.

Psoriatic arthritis (PsA) patients have been shown to have a discontinuation rate with biologic and nonbiologic psoriasis treatments ranging from $60 \%-80 \%$.

In a recent study, patients who switched during the 1-year posttreatment period incurred significantly higher costs compared with nonswitchers, including overall health care ( $\$ 44,622$ vs. $\$ 35,613)$, overall medical ( $\$ 8,282$ vs. $\$ 4,597)$, and psoriasisrelated health care costs $(\$ 35,760$ vs. $\$ 29,453)$.

\section{What this study adds}

Moderate to severe psoriasis and/or PsA patients who were nonpersistent to a biologic incurred significantly higher overall medical costs compared with persistent patients, with ambulatory utilization being the primary driver of the cost burden.

Approximately $40 \%$ of the total overall medical costs were attributed to psoriasis and/or PsA overall.

Owing to increased biologic use and the cost of biologics per unit, persistent patients were seen to incur significantly higher pharmacy utilization and costs in the sample. Total costs combining medical and pharmacy costs were similar between the persistent and nonpersistent patients. 
$\mathrm{P}$ soriasis is a chronic, hyper-proliferative dermatological condition that can also present with arthritic symptoms known as psoriatic arthritis (PsA). ${ }^{1}$ Psoriasis has an estimated prevalence of $1.4 \%-2.5 \%$ among children, adolescents, and adults in the United States. ${ }^{2}$ Coexisting immune-mediated skin disorders have physical and psychosocial consequences, affecting work productivity and overall quality of life. ${ }^{3}$

The disease presents a clinical and economic burden on society. A recent systematic review suggested that total direct costs per year attributed to psoriasis are $\$ 51.7-\$ 63.2$ billion, with medical comorbidities contributing as much as $\$ 36.4$ billion. ${ }^{4}$ Another study suggested that moderate to severe psoriasis and/or PsA patients had 5 times higher costs and significantly higher likelihood of inpatient admissions, emergency department (ED) visits, and outpatient visits than patients without psoriasis or PsA. ${ }^{5}$

With the advent of biologics, the economic burden of patients with moderate to severe psoriasis and/or PsA has reduced significantly. ${ }^{6}$ However, recent literature has demonstrated that there is a $46 \%$ discontinuation rate among biologic users, which has significantly affected the health outcomes of psoriatic patients, ${ }^{7}$ and a recent study revealed that, on average, 1 treatment change occurred among patients during the 1-year post-treatment period. ${ }^{8}$ A recent study using a U.S. diseasebased registry showed that persistence to tumor necrosis factor (TNF)-inhibiting biologics dropped from $83.2 \%$ at 6 months to as low as $36.1 \%$ at the end of 2 years post-treatment initiation. ${ }^{9}$ In addition, another study claimed that discontinuation rates among psoriasis patients who initiated nonbiologic oral drugs or biologics ranged between $60 \%$ and $80 \% .{ }^{10}$

Treatment patterns are likely to have an effect on overall treatment costs among psoriasis patients. A recent research study claimed that those who switched during their initial treatment were associated with significantly greater overall costs $(\$ 44,622$ vs. $\$ 35,613)$ and psoriasis-related health care costs ( $\$ 35,760$ vs. $\$ 29,453)$ compared with nonswitchers. ${ }^{11}$

Treatment persistence among prevalent biologic users has not been explored to its full potential, and there is a dearth of evidence regarding the economic burden among prevalent moderate to severe psoriasis and/or PsA patients who are persistent on 1 psoriasis- and/or PsA-related biologic versus those who switch to different biologics or discontinue use of any biologic. Therefore, this retrospective cohort study examined overall and moderate to severe psoriasis- and/or PsA-related health care utilization and costs among persistent versus nonpersistent patients using the U.S. Department of Defense (DoD) database.

\section{Methods}

\section{Study Design and Data Source}

This was a retrospective cohort claims analysis using medical, pharmacy, and enrollment information from the U.S. DoD database for the period of November 1, 2010-November 30, 2015. This study examined health care costs and utilization between patients who were persistent and nonpersistent to index biologic among patients with moderate to severe psoriasis and/or PsA.

The U.S. DoD health care system uses the largest computable electronic health record system in the United States to provide health care to over 9.5 million beneficiaries located all over the world. This data repository includes comprehensive datasets providing integrated information about inpatient, outpatient, ED, and pharmacy claims from U.S. DoD facilities and civilian/private sector care for eligible beneficiaries. Full costs were captured for health care services provided in military treatment facilities including direct costs, clinician salary, and costs of surgical and ancillary procedures. Total amount paid by TRICARE, total patient cost share, and total other health insurance amount paid were captured for health care services provided at civilian facilities. Because this study did not make use of personally identifiable information, institutional review board approval was not needed.

\section{Patient Selection}

Adult patients (aged $\geq 18$ years) were included if they had $\geq 2$ diagnosis claims for psoriasis (International Classification of Diseases, Ninth Revision, Clinical Modification [ICD-9-CM] code 696.1; International Classification of Diseases, Tenth Revision, Clinical Modification [ICD-10-CM] codes L40.0, L40.1, L40.2, L40.3, L40.4, and L40.8) or PsA (ICD-9-CM code 696.0; ICD$10-\mathrm{CM}$ codes L40.54 and L40.59) during the study period (November 1, 2010-October 1, 2015). The first psoriasis or PsA diagnosis claim date during the index period (November 1 , 2011-November 30, 2014) was designated as the index diagnosis date. Patients were required to have continuous health plan enrollment for $\geq 12$ months pre-index date (baseline period) and post-index date (follow-up period). Those who had $\geq 1$ nontopical systemic therapy (including biologics and nonbiologics) or phototherapy (Appendix A, available in online article) received within 30 days after index date or during the 12-month baseline period were considered moderate to severe patients. ${ }^{12,13}$ In this study, patients who had biologic use of etanercept, adalimumab, ustekinumab, or infliximab within 30 days after or on the index date were examined among the overall prevalent moderate to severe patients.

\section{Cohort Assignment}

The first occurrence of prevalent biologic use of etanercept, adalimumab, ustekinumab, or infliximab on or within 30 days after the index diagnosis was defined as the index biologic. Healthcare Common Procedure Coding System (HCPCS) codes and National Drug Code (NDC) numbers were used to identify biologic drugs (Appendix A). 
The persistent and nonpersistent cohorts were created based on treatment persistence of index biologic use in the l-year post-index period. Persistence to index therapy was determined based on a 90-day gap and days supply defined according to each biologic's dosing schedule: etanercept, 7 days; adalimumab, 14 days; ustekinumab, 90 days; and infliximab, 60 days.

If there was a gap in the administration of the index biologic $>90$ days beyond the days supply period of the previous injection, the patient was assigned to the nonpersistent cohort; otherwise, the patient was considered persistent to their index biologic. Patients who switched to a different biologic or discontinued any biologic use were considered as nonpersistent patients. Persistent versus nonpersistent patients (based on the classification above) were compared for the outcomes described in the "Results" section.

\section{Study Variables}

Baseline Patient Characteristics. Baseline demographic and clinical characteristics during the 12 months prior to the index date were measured. Baseline characteristics included demographics (age, sex, U.S. geographic region), clinical characteristics (Charlson Comorbidity Index [CCI] score and individual comorbid conditions identified via ICD-9-CM and ICD-10-CM codes; see Appendix B, available in online article). In addition, baseline biologic, nonbiologic, and other medication use (anti-anxiety, antidepressants, etc.) and baseline health care utilization and costs were examined. Patients' disease status including psoriasis only, PsA only, or both psoriasis and PsA was examined during the 12-month baseline period and on the index date.

Outcome Variables. Overall and moderate to severe psoriasisand/or PsA-related health care utilization and costs were measured over the entire 12-month follow-up period. The number and percentage of patients in each cohort utilizing health care services (number of inpatient admissions and number of ambulatory, ED, and pharmacy visits) were calculated for the 1-year follow-up period. Disease-related utilizations and costs were calculated using the claims with primary or secondary psoriasis and/or PsA diagnosis or any psoriasis and/or PsA treatment (Appendix A). Costs were adjusted to 2015 U.S. dollars using the medical care component of the Consumer Price Index. Specifically, the calculated cost categories included inpatient visit, ambulatory visit, ED visit, pharmacy, total medical (inpatient + ambulatory+ED), and total costs (inpatient + ambulatory $+\mathrm{ED}+$ pharmacy).

\section{Statistical Analysis}

Baseline and outcome variables were analyzed descriptively. Numbers and percentages were provided for categorical variables. Means and standard deviations were provided for continuous variables. For categorical variables, $P$ values were calculated according to the chi-square test; for continuous variables, t-tests were used to calculate $P$ values. T-tests and Pearson chi-squared tests were used to test statistically significant differences at the 5\% level for continuous and categorical variables, respectively. Standardized differences (STDs) were calculated to distinguish practical from statistical significance and were reported as 100 times the absolute value of the actual STD to allow for easy interpretation. STDs with a value $>10$ were considered significant.

For multivariate analysis, generalized linear models were used to evaluate health care utilization and costs adjusting for patient demographics (age, gender, and region), baseline clinical characteristics (CCI, diabetes, obesity, overweight, dyslipidemia, hypertension, coronary artery disease, number of phototherapies, biologics and nonbiologics, baseline use of disease-modifying antirheumatic drug, statins, and anti-anxiety medications), baseline health care utilization, and disease status (psoriasis only, PsA only, or both psoriasis and PsA). Negative binomial distribution and a log-link function for the number of visits and gamma distribution with a log-link function were applied for health care costs. ${ }^{14,15}$ Adjusted means after controlling patient demographic and clinical characteristics were calculated for the persistent and nonpersistent cohorts.

All analyses were carried out using SAS version 9.3 (SAS Institute, Cary, NC).

\section{Results}

\section{Patient Demographic and Clinical Characteristics}

A total of 2,945 patients had etanercept, adalimumab, infliximab, or ustekinumab within 30 days of the index diagnosis; of these patients, 1,899 (64.5\%) were persistent and 1,046 (35.5\%) were nonpersistent to their index biologic drug during the 1-year follow-up period. In the persistent cohort, 913 (48.1\%) patients had a psoriasis diagnosis only, 470 (24.7\%) had PsA diagnosis only, and 516 (27.2\%) had both psoriasis and PsA, while in the nonpersistent cohort, 594 (56.8\%) had psoriasis only, 226 (21.6\%) had PsA only, and 226 (21.6\%) had psoriasis and PsA during the 12-month pre-index period and on the index date (Figure 1).

Persistent patients with moderate to severe psoriasis and/or PsA were older (49.2 vs. 45.5 years; $P<0.001)$ and predominantly male ( $52.1 \%$ vs. $45.0 \%$; $P<0.001)$ compared with nonpersistent patients. Both cohorts had a similar comorbidity index score of 0.7 at baseline. Persistent patients were more likely to have dyslipidemia ( $40.4 \%$ vs. $34.7 \%$; $P=0.002$ ) compared with nonpersistent patients. Other frequent comorbidities included hypertension, diabetes, and obesity, with similar distribution between the 2 cohorts. The persistent cohort was found to have a significantly higher mean number for biologic injections (6.3 vs. $4.7 ; P<0.001)$ and was more likely to have statin use $(23.3 \%$ vs. $18.4 \%$; $P=0.002)$ but less likely to have 


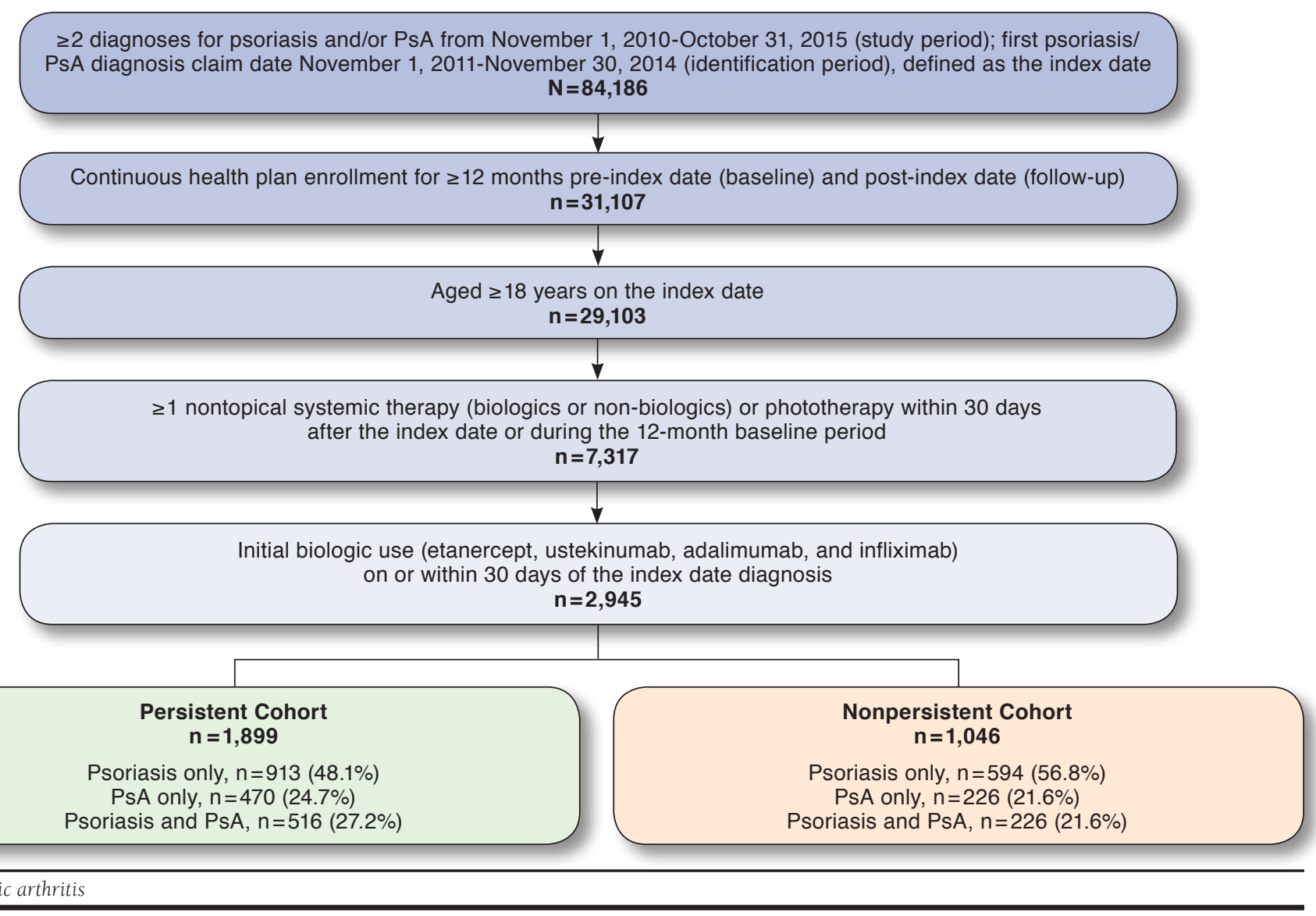

anxiolytic use $(29.5 \%$ vs. $36.8 \%, P<0.001)$ at baseline compared with the nonpersistent cohort (Table 1).

\section{Outcomes}

Overall Health Care Utilization and Costs. During the 1-year post-index period, nonpersistent patients were found to have higher unadjusted health care resource utilization compared with persistent patients for inpatient admissions (0.24 vs. 0.13; $P<0.001$ ), ambulatory visits (26.1 vs. 22.6; $P<0.001)$, and ED visits (0.8 vs. $0.5 ; P<0.001)$ but a lower number of pharmacy visits ( 22.4 vs. $24.1 ; P=0.005$ ). Thus, total medical costs were higher for nonpersistent patients compared with persistent patients $(\$ 13,957$ vs. $\$ 11,827 ; P=0.007)$, of which $75 \%-85 \%$ of the costs were contributed by ambulatory use. The total overall costs were similar between the 2 cohorts (Table 2).

After adjusting for baseline demographics and clinical characteristics, persistent patients had fewer overall inpatient admissions ( 0.1 vs. $0.2 ; P<0.001)$, fewer ambulatory visits $(21.4$ vs. 23.9; $P=0.007$ ), fewer ED visits ( 0.4 vs. $0.6 ; P<0.001)$, and similar pharmacy visits (22.3 vs. 20.7; $P=0.068)$ compared with nonpersistent patients (Figure 2). Consequently, persistent patients had significantly lower overall inpatient (\$951 vs. $\$ 1,969 ; P=0.003)$, ambulatory ( $\$ 7,349$ vs. $\$ 9,646 ; P<0.001)$, ED ( $\$ 294$ vs. $\$ 448 ; P<0.001)$, and total medical costs $(\$ 8,964$ vs. $\$ 12,457 ; P<0.001)$ compared with nonpersistent patients. However, overall pharmacy costs per patient were significantly higher in the persistent cohort compared with the nonpersistent cohort $(\$ 10,684$ vs. $\$ 7,849 ; P<0.001)$. Total overall costs combining medical and pharmacy costs were similar between the cohorts $(\$ 22,678$ vs. $\$ 21,477 ; P=0.122$; Figure 3$)$.

Moderate to Severe Psoriasis- and/or PsA-Related Health Care Utilization and Costs. During the 1-year follow-up period, persistent patients had significantly fewer unadjusted psoriasis- and/or PsA-related inpatient admissions but more pharmacy visits. Health care costs related to psoriasis and/or PsA were significantly higher for ambulatory visits, pharmacy, and total costs before adjustment (Table 2).

After adjusting for patient demographic and clinical characteristics, persistent patients had a significantly lower number 


\section{Evaluating the Effect of Treatment Persistence on the Economic Burden of Moderate to Severe Psoriasis and/or Psoriatic Arthritis Patients in the U.S. Department of Defense Population}

\section{TABLE 1 Baseline Characteristics of Persistent Cohort Versus Nonpersistent Cohort in the}

U.S. DoD Population

\begin{tabular}{|c|c|c|c|c|c|c|}
\hline \multirow[b]{2}{*}{ Baseline Characteristics } & \multicolumn{2}{|c|}{$\begin{array}{c}\begin{array}{c}\text { Nonpersistent Patients } \\
\mathbf{n}=1,046\end{array} \\
\end{array}$} & \multicolumn{4}{|c|}{$\begin{array}{c}\text { Persistent Patients } \\
\mathrm{n}=1,899\end{array}$} \\
\hline & N/Mean & $\% / S D$ & N/Mean & $\% / S D$ & $P$ Value & STD \\
\hline Age, mean & 45.5 & 12.6 & 49.2 & 11.5 & $<0.001^{\mathrm{a}}$ & $31.31^{\mathrm{b}}$ \\
\hline \multicolumn{7}{|l|}{ Age group, years, n (\%) } \\
\hline $18-40$ & 392 & 37.5 & 426 & 22.4 & $<0.001^{\mathrm{a}}$ & $33.28^{b}$ \\
\hline $41-64$ & 619 & 59.2 & 1,373 & 72.3 & $<0.001^{\mathrm{a}}$ & $27.91^{b}$ \\
\hline $65+$ & 35 & 3.3 & 100 & 5.3 & $0.017^{\mathrm{a}}$ & 9.47 \\
\hline \multicolumn{7}{|l|}{ Sex, n (\%) } \\
\hline Male & 471 & 45.0 & 990 & 52.1 & $<0.001^{\mathrm{a}}$ & $14.24 \mathrm{~b}$ \\
\hline \multicolumn{7}{|l|}{ U.S. geographic region, $n(\%)$} \\
\hline Northeast & 45 & 4.3 & 61 & 3.2 & 0.129 & 5.73 \\
\hline $\begin{array}{l}\text { North Central } \\
\end{array}$ & 78 & 7.5 & 179 & 9.4 & 0.070 & 7.08 \\
\hline South & 653 & 62.4 & 1,143 & 60.2 & 0.233 & 4.6 \\
\hline West & 238 & 22.8 & 460 & 24.2 & 0.369 & 3.47 \\
\hline Overseas & 10 & 1.0 & 17 & 0.9 & 0.868 & 0.63 \\
\hline Unknown & 22 & 2.1 & 39 & 2.1 & 0.928 & 0.35 \\
\hline Baseline CCI, mean (SD) & 0.7 & 1.2 & 0.7 & 1.1 & 0.862 & 0.68 \\
\hline $0, \mathrm{n}(\%)$ & 633 & 60.5 & 1,122 & 59.1 & 0.448 & 2.92 \\
\hline $1, \mathrm{n}(\%)$ & 266 & 25.4 & 483 & 25.4 & 0.998 & 0.01 \\
\hline $2+, \mathrm{n}(\%)$ & 147 & 14.1 & 294 & 15.5 & 0.299 & 4.03 \\
\hline \multicolumn{7}{|l|}{ Baseline individual comorbid conditions, $\mathbf{n}(\%)$} \\
\hline Diabetes mellitus & 149 & 14.2 & 286 & 15.1 & 0.550 & 2.31 \\
\hline Inflammatory bowel disease & 42 & 4.0 & 67 & 3.5 & 0.503 & 2.56 \\
\hline Multiple sclerosis & 4 & 0.4 & 6 & 0.3 & 0.767 & 1.13 \\
\hline Ankylosing spondylitis and axial ankylosing spondylitis & 31 & 3.0 & 51 & 2.7 & 0.661 & 1.68 \\
\hline Obesity & 107 & 10.2 & 190 & 10.0 & 0.847 & 0.74 \\
\hline Overweight & 35 & 3.3 & 49 & 2.6 & 0.232 & 4.52 \\
\hline Arteriosclerotic vascular disease & 2 & 0.2 & 7 & 0.4 & 0.404 & 3.36 \\
\hline Cerebrovascular ischemia & 24 & 2.3 & 28 & 1.5 & 0.106 & 6.03 \\
\hline Peripheral vascular disease & 5 & 0.5 & 19 & 1.0 & 0.131 & 6.1 \\
\hline Atherosclerosis & 18 & 1.7 & 18 & 0.9 & 0.068 & 6.74 \\
\hline Dyslipidemia & 363 & 34.7 & 768 & 40.4 & $0.002^{\mathrm{a}}$ & $11.87^{\mathrm{b}}$ \\
\hline Hypertension & 377 & 36.0 & 752 & 39.6 & 0.057 & 7.34 \\
\hline Stroke & 4 & 0.4 & 8 & 0.4 & 0.874 & 0.61 \\
\hline Myocardial infarction & 9 & 0.9 & 24 & 1.3 & 0.320 & 3.93 \\
\hline Coronary artery disease & 50 & 4.8 & 98 & 5.2 & 0.651 & 1.75 \\
\hline \multicolumn{7}{|l|}{ Baseline psoriasis/PsA treatment, mean (SD) } \\
\hline Number of phototherapy treatments & 0.5 & 3.5 & 0.5 & 4.4 & 0.962 & 0.18 \\
\hline Number of biologics & 4.7 & 3.6 & 6.3 & 3.8 & $<0.001^{\mathrm{a}}$ & $42.81^{b}$ \\
\hline Number of nonbiologics & 1.4 & 3.4 & 1.4 & 2.9 & 0.882 & 0.58 \\
\hline \multicolumn{7}{|l|}{ Baseline medication use, $\mathrm{n}(\%)$} \\
\hline Antidepressant & 287 & 27.4 & 445 & 23.4 & $0.016^{\mathrm{a}}$ & 9.2 \\
\hline Anxiolytic & 385 & 36.8 & 561 & 29.5 & $<0.001^{\mathrm{a}}$ & $15.47^{\mathrm{b}}$ \\
\hline Antidiabetic & 137 & 13.1 & 274 & 14.4 & 0.319 & 3.86 \\
\hline Angiotensin II receptor antagonist & 110 & 10.5 & 211 & 11.1 & 0.620 & 1.91 \\
\hline Beta blocker & 140 & 13.4 & 285 & 15.0 & 0.230 & 4.65 \\
\hline Disease-modifying antirheumatic drug & 287 & 27.4 & 517 & 27.2 & 0.901 & 0.48 \\
\hline Statin & 192 & 18.4 & 443 & 23.3 & $0.002^{\mathrm{a}}$ & $12.26^{\mathrm{b}}$ \\
\hline
\end{tabular}

aStatistical significance.

bSignificant difference. Standardized differences were reported as 100 times the absolute value of the actual standardized difference to allow for easy interpretation Standardized differences $>10$ were considered significant.

$C C I=$ Charlson Comorbidity Index; DoD = Department of Defense; $P S A=$ ssoriatic arthritis; $S D=$ standard deviation; STD = standardized difference. 


\section{Evaluating the Effect of Treatment Persistence on the Economic Burden of Moderate to Severe Psoriasis and/or Psoriatic Arthritis Patients in the U.S. Department of Defense Population}

\section{TABLE 2 Follow-up Unadjusted Health Care Utilization and Costs Among Persistent and Nonpersistent}

Patients in the U.S. DoD Population

\begin{tabular}{|c|c|c|c|c|c|c|}
\hline \multirow[b]{2}{*}{ Follow-up Health Care Utilization and Costs } & \multicolumn{2}{|c|}{$\begin{array}{c}\begin{array}{c}\text { Nonpersistent Patients } \\
\mathrm{n}=1,046\end{array} \\
\end{array}$} & \multicolumn{4}{|c|}{$\begin{array}{c}\text { Persistent Patients } \\
\mathrm{n}=1,899\end{array}$} \\
\hline & N/Mean & $\% / S D$ & N/Mean & $\% / S D$ & $P$ Value & STD \\
\hline \multicolumn{7}{|l|}{ Follow-up overall health care utilization, mean (SD) } \\
\hline Number of inpatient admissions & 0.2 & 0.7 & 0.1 & 0.5 & $<0.001^{\mathrm{a}}$ & $16.89^{b}$ \\
\hline Number of ambulatory visits & 26.1 & 23.4 & 22.6 & 18.8 & $<0.001^{\mathrm{a}}$ & $16.35^{\mathrm{b}}$ \\
\hline Number of ED visits & 0.8 & 1.5 & 0.5 & 1.2 & $<0.001^{\mathrm{a}}$ & $21.55^{\mathrm{b}}$ \\
\hline Number of pharmacy visits & 22.4 & 16.5 & 24.1 & 15.1 & $0.005^{\mathrm{a}}$ & $10.86^{b}$ \\
\hline \multicolumn{7}{|l|}{ Follow-up overall health care costs, mean (SD) } \\
\hline Inpatient costs & $\$ 2,915$ & $\$ 11,591$ & $\$ 1,368$ & $\$ 5,943$ & $<0.001^{\mathrm{a}}$ & $16.80^{\mathrm{b}}$ \\
\hline Ambulatory costs & $\$ 10,445$ & $\$ 14,701$ & $\$ 10,113$ & $\$ 14,720$ & 0.558 & 2.26 \\
\hline ED costs & $\$ 596$ & $\$ 1,349$ & $\$ 346$ & $\$ 958$ & $<0.001^{\mathrm{a}}$ & $21.42^{\mathrm{b}}$ \\
\hline Pharmacy costs & $\$ 8,736$ & $\$ 12,896$ & $\$ 12,632$ & $\$ 15,252$ & $<0.001^{\mathrm{a}}$ & $27.59 \mathrm{~b}$ \\
\hline Total medical costs (inpatient + ambulatory + ED) & $\$ 13,957$ & $\$ 22,214$ & $\$ 11,827$ & $\$ 17,063$ & $0.007^{\mathrm{a}}$ & $10.75^{b}$ \\
\hline Total costs (inpatient + ambulatory + ED + pharmacy) & $\$ 22,693$ & $\$ 25,504$ & $\$ 24,459$ & $\$ 20,760$ & 0.055 & 7.60 \\
\hline \multicolumn{7}{|c|}{ Follow-up moderate to severe psoriasis- and/or PsA-related health care utilization, mean (SD) } \\
\hline Number of inpatient admissions & 0.1 & 0.4 & 0.1 & 0.3 & $0.002^{\mathrm{a}}$ & $12.24 \mathrm{~b}$ \\
\hline Number of ambulatory visits & 6.7 & 7.7 & 6.6 & 6.4 & 0.681 & 1.62 \\
\hline Number of ED visits & 0.1 & 0.3 & 0.0 & 0.3 & 0.148 & 5.53 \\
\hline Number of pharmacy visits & 5.0 & 3.8 & 8.2 & 4.4 & $<0.001^{\mathrm{a}}$ & $80.20^{\mathrm{b}}$ \\
\hline \multicolumn{7}{|c|}{ Follow-up moderate to severe psoriasis- and/or PsA-related health care costs, mean (SD) } \\
\hline Inpatient costs & $\$ 980$ & $\$ 5,068$ & $\$ 674$ & $\$ 4,389$ & 0.100 & 6.46 \\
\hline Ambulatory costs & $\$ 4,435$ & $\$ 8,710$ & $\$ 5,610$ & $\$ 12,289$ & $0.003^{a}$ & $11.03^{\mathrm{b}}$ \\
\hline ED costs & $\$ 33$ & $\$ 207$ & $\$ 25$ & $\$ 224$ & 0.330 & 3.71 \\
\hline Pharmacy costs & $\$ 6,097$ & $\$ 8,770$ & $\$ 10,669$ & $\$ 13,337$ & $<0.001^{\mathrm{a}}$ & $40.51^{b}$ \\
\hline Total medical costs (inpatient + ambulatory + ED) & $\$ 5,448$ & $\$ 10,297$ & $\$ 6,309$ & $\$ 13,114$ & $0.050^{\mathrm{a}}$ & 7.30 \\
\hline Total costs (inpatient + ambulatory + ED + pharmacy) & $\$ 11,545$ & $\$ 12,840$ & $\$ 16,977$ & $\$ 15,803$ & $<0.001^{\mathrm{a}}$ & $37.73^{b}$ \\
\hline \multicolumn{7}{|c|}{ Follow-up drug-specific utilization for nontopical systemic therapy, n (\%) } \\
\hline \multicolumn{7}{|l|}{ Any prescription (yes/no) } \\
\hline Use of oral prescriptions (yes/no) & 66 & 6.3 & 94 & 4.9 & 0.119 & 5.90 \\
\hline Use of injections (yes/no) & 1,014 & 96.9 & 1,899 & 100.0 & $<0.001^{\mathrm{a}}$ & $25.11^{\mathrm{b}}$ \\
\hline \multicolumn{7}{|l|}{ Number of oral prescriptions/injections, mean (SD) } \\
\hline Number of oral prescriptions & 0.2 & 1.2 & 0.2 & 1.2 & 0.751 & 1.23 \\
\hline Number of injections & 5.6 & 4.1 & 9.3 & 4.2 & $<0.001^{\mathrm{a}}$ & $90.80^{\mathrm{b}}$ \\
\hline
\end{tabular}

of psoriasis- and/or PsA-related inpatient admissions (0.05 vs. $0.08 ; P=0.001$ ) but a higher number of pharmacy visits for psoriasis and/or PsA treatment (7.6 vs. 4.8; $P<0.001)$. Similar outcomes were observed for psoriasis- and/or PsA-related ambulatory or ED visits (Figure 2). Of the total overall medical costs, almost $38 \%-40 \%$ of costs were attributed to moderate to severe psoriasis and/or PsA. Specifically, nonpersistent patients incurred significantly greater mean psoriasis- and/or PsArelated total medical costs than persistent patients per patient $(\$ 5,041$ vs. $\$ 3,395 ; P<0.001)$, with ambulatory use being the driver of these costs. However, average psoriasis-related pharmacy costs were significantly greater in the persistent cohort ( $\$ 7,668$ vs. $\$ 5,171 ; P<0.001$; Figure 3).

\section{Discussion}

Our analysis was conducted from a large, comprehensive, administrative national DoD dataset that offers a large sample of military personnel and their dependents to evaluate the association between persistence to first occurring prevalent biologic medication used and overall and psoriasis-related health care utilization and costs. The DoD population is composed of a diverse population, with $14 \%$ on active duty and the remaining $86 \%$ consisting of dependents and retirees. ${ }^{16}$ In addition, $78 \%$ of the DoD population is aged $\geq 18$ years compared with $75 \%$ of the general U.S. population, which may add to the external validity of this analysis. ${ }^{16}$ To the best of 
FIGURE 2 Adjusted Overall and Psoriasis- and/or PsA-Related Health Care Utilization Among Persistent and Nonpersistent Patients in the U.S. DoD Population

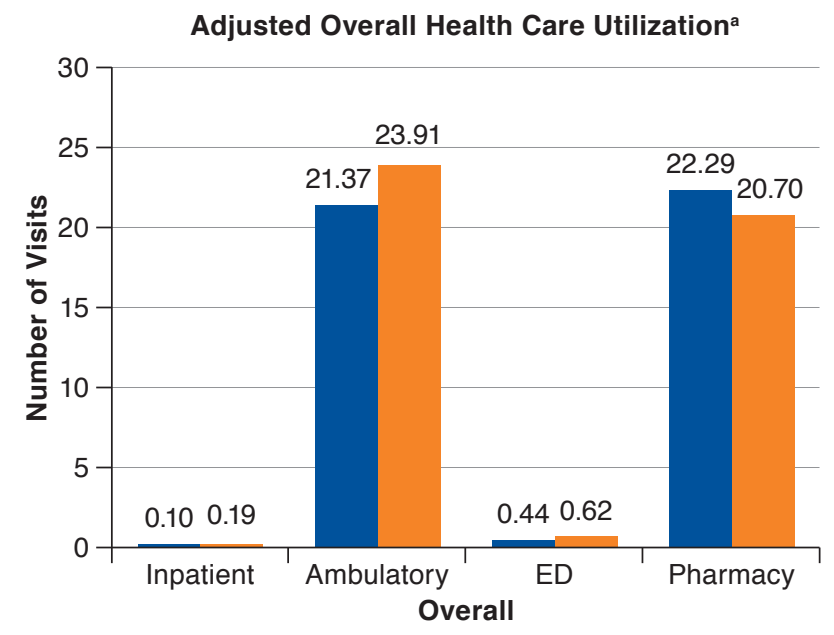

Persistent $(n=1,899) \quad$ Nonpersistent $(n=1,046)$
Adjusted Psoriasis- and/or PsA-Related Health Care Utilization ${ }^{b}$

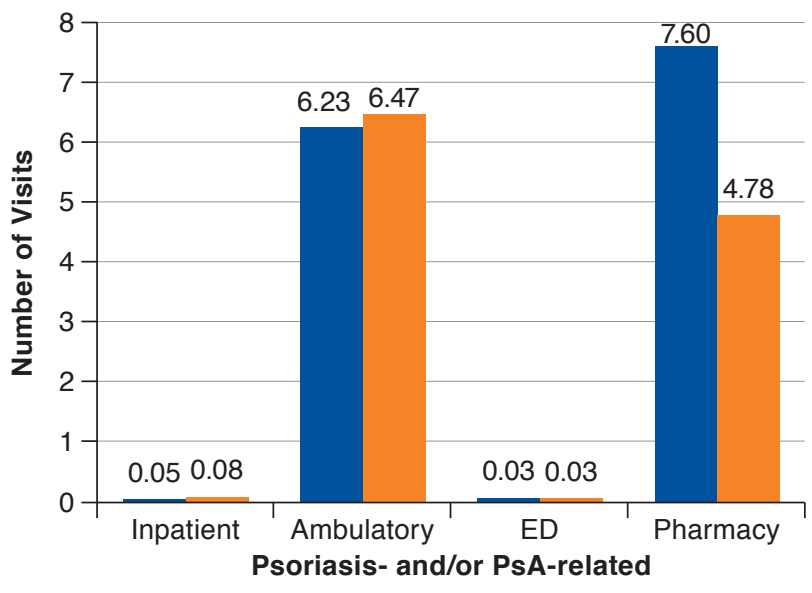

Persistent $(n=1,899) \quad$ Nonpersistent $(n=1,046)$

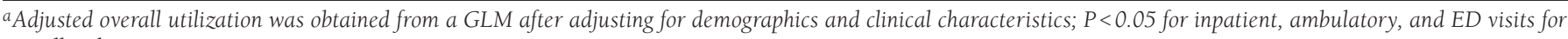
overall utilization.

${ }^{b}$ Adjusted psoriasis and/or PsA-related utilization was obtained from a GLM after adjusting for demographics and clinical characteristics; P $<0.05$ for inpatient and pharmacy visits for psoriasis- and/or PsA-related utilization.

DoD=Department of Defense; ED=emergency department; GLM=generalized linear model; PsA=psoriatic arthritis.

our knowledge, this is the first economic analysis conducted among DoD enrollees for the psoriasis population.

Prior research has shown that more than $50 \%$ of physicians prescribe systemic biologics for the treatment of psoriasis..$^{17,18}$ However, the economic burden of psoriasis due to biologic therapy among moderate to severe psoriasis and/or PsA patients is escalating, as is indicated in the literature. ${ }^{19}$

A recent study showed that almost $60 \%$ of biologic users among psoriasis patients experienced a therapy change within 1 year of drug use, with a mean time to therapy change of close to 4 months. ${ }^{20}$ In our study, we observed that most psoriasis and/or PsA patients were persistent to biologics (64.5\%), but a significant proportion remained nonpersistent (35.5\%). This shows that the need for sufficient and effective treatment for psoriasis may be unmet due to lack of treatment persistence. Dermatology clinic patients who discontinue their treatments have higher Psoriasis Area and Severity Index (PASI) scores compared with those who persisted on their initial biologic drug. ${ }^{21}$ Therefore, the lack of optimal biologic persistence may be correlated with severe disease and is likely to be associated with reduced treatment effectiveness.

More than $80 \%$ of psoriasis patients were found to have psychiatric comorbidities and, therefore, we discussed depression and anxiety as part of our analysis. ${ }^{22,23}$ We found that at baseline, more nonpersistent patients had antidepressant (27.4\% vs. $23.4 \%)$ and anti-anxiety (36.8\% vs. $29.5 \%)$ drug use compared with persistent patients. This strongly aligns with antidepressant prevalence estimates obtained in the literature among psoriasis patients of 17\%-33\% $\%^{24-26}$ and anxiolytic prevalence estimates of 20\%-30\%. ${ }^{27}$ This also may likely be one of the drivers of nonpersistence in our study as has been hypothesized by researchers in the past. ${ }^{28,29}$

Treatment nonpersistence may lead to treatment failure due to discontinuation/reinitiation or biologic switch during the course of treatment..$^{30}$ In the literature, this has been hypothesized to result in increased health care costs due to costs of reinitiation. A recent study demonstrated that treatment regimen failures had significant overall health care costs among psoriasis patients in the 12-month baseline period; specifically, the treatment regimen failure cohort incurred $\$ 8,024$ per patient compared with $\$ 6,637$ per patient. ${ }^{31}$ In our multivariate analysis, we found that nonpersistent patients had significantly higher mean overall total medical costs per patient compared with persistent patients $(\$ 12,457$ vs. $\$ 8,964)$ in the follow-up period, of which, approximately $40 \%$ of the costs were attributed to moderate to severe psoriasis and/or PsA diagnoses. Therefore, of the total overall burden, $40 \%$ of the costs were attributed to moderate to severe psoriasis and/or PsA, which is 
FIGURE 3 Adjusted Overall and Psoriasis- and/or PsA-Related Health Care Costs Among Persistent and Nonpersistent Patients in the U.S. DoD Population (12 Months of Follow-up)

Adjusted Overall Costs ${ }^{a}$

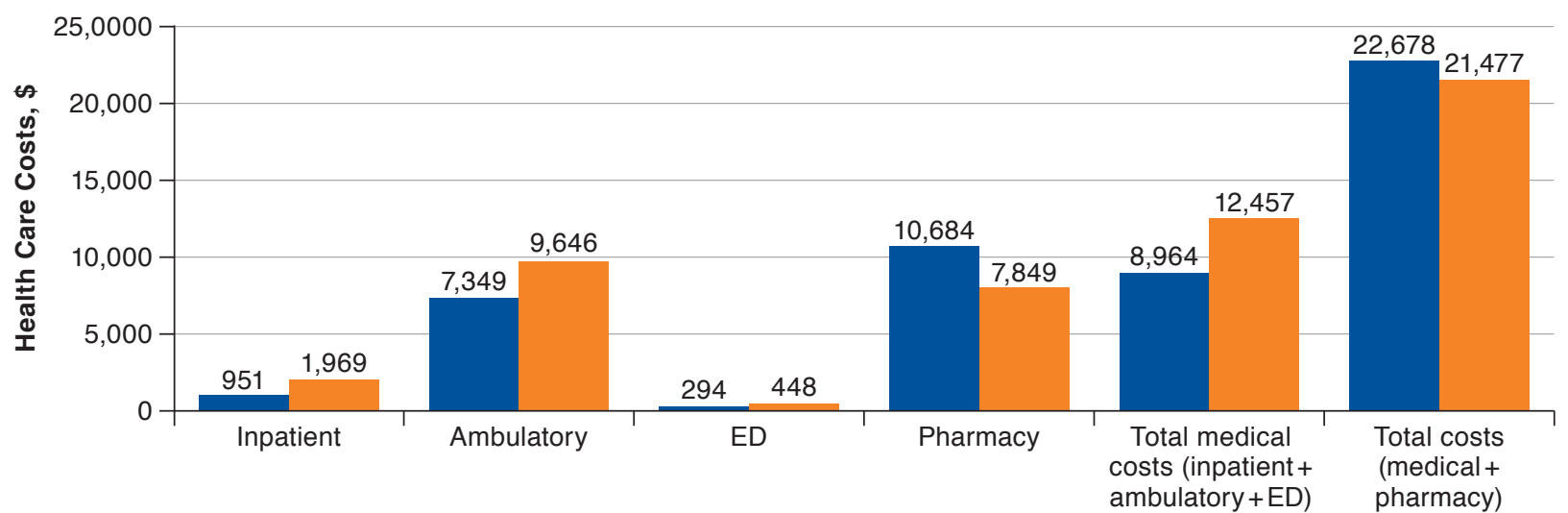

Persistent $(n=1,899) \quad$ Nonpersistent $(n=1,046)$

Adjusted Psoriasis- and/or PsA-Related Costs ${ }^{b}$

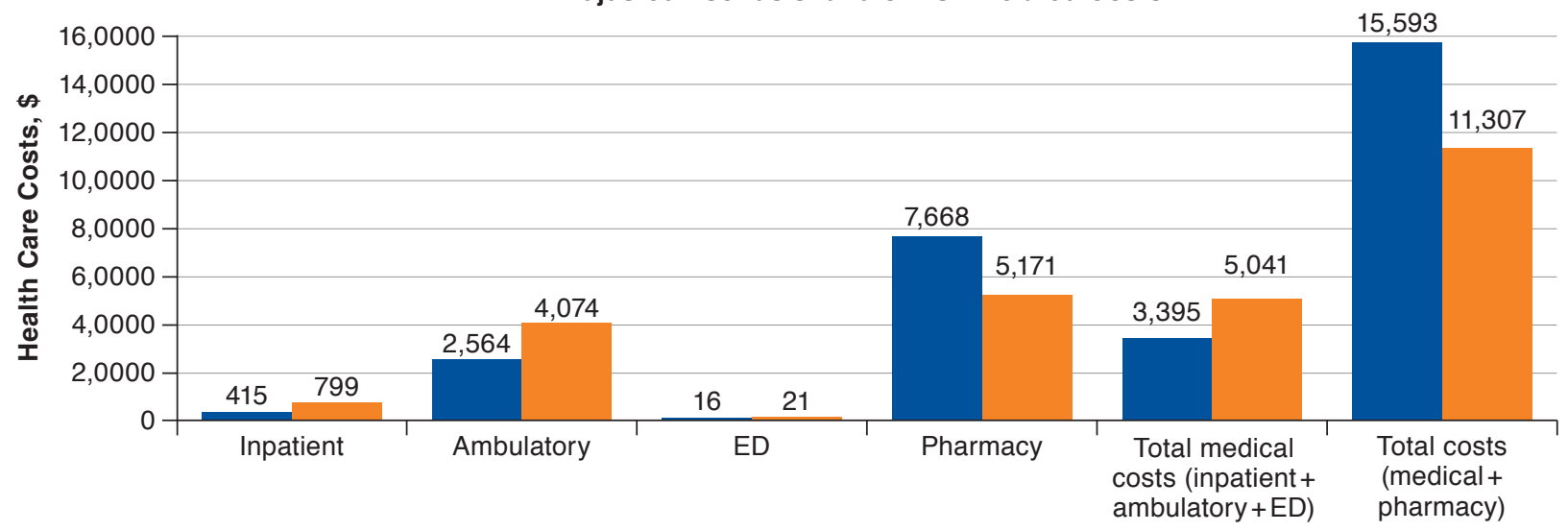

Persistent $(n=1,899) \quad$ Nonpersistent $(n=1,046)$

aAdjusted overall costs were obtained from a GLM after adjusting for demographics and clinical characteristics; $P<0.05$ for inpatient, ambulatory, ED, pharmacy, and medical costs for overall costs.

${ }^{b}$ Adjusted psoriasis and/or PSA-related costs were obtained from a GLM after adjusting for demographics and clinical characteristics; $P<0.05$ for inpatient, ambulatory, pharmacy, medical, and total costs for psoriasis and/or PsA-related costs.

DoD=Department of Defense; $E D=$ emergency department; $G L M=$ generalized linear model; PsA = psoriatic arthritis; U.S. =United States.

quite significant. In addition, nonpersistent patients had higher mean psoriasis- and/or PsA-related total medical costs per patient $(\$ 5,041$ vs. $\$ 3,395)$ compared with persistent patients. This strongly aligns with the general hypothesis that treatment nonpersistence may be an important predictor of increased overall and psoriasis-related costs.

Studies suggest that lack of persistence can play a role in increasing treatment costs in psoriasis therapies. A recent study conducted among U.S. veterans showed that persistence on biologic treatment was approximately $50 \%$ over a 1-year period and accumulated average drug and administration costs (combined) in the range of $\$ 15,000$ - $\$ 19,000$ per patient per year. ${ }^{32}$ In our study, persistent patients had a significantly higher mean number of biologic injections compared with nonpersistent patients during follow-up (9.3 vs. 5.6; $P<0.001$; Table 2). Our multivariate model showed that after adjusting for baseline biologic use, persistent patients accumulated significantly higher mean moderate to severe psoriasis- and/or PsA-related pharmacy costs per patient over the 1-year follow-up $(\$ 7,668$ vs. $\$ 5,171 ; P<0.001)$, owing to high biologic use. This can be a 


\section{Evaluating the Effect of Treatment Persistence on the Economic Burden of Moderate to Severe Psoriasis and/or Psoriatic Arthritis Patients in the U.S. Department of Defense Population}

result of increased biologic utilization and the associated high costs for biologics during the follow-up period. Having said the above, it should be noted that these higher costs were offset by lower overall and psoriasis-related medical costs when compared with nonpersistent patients.

Although reasons of nonpersistence cannot be conclusively stated, cohorts may have discontinued, switched, or restarted the index biologic. Likewise, such patient behavior leading to nonpersistence among patients may have led to greater total medical costs.

\section{Limitations}

Our analysis had several limitations. First, there is potential for measurement errors, as it is possible that some ICD-9-CM or ICD-10-CM codes may have been incorrectly recorded, misused, or not entered at all. Second, in studies using administrative claims data, the true validity of the variables analyzed in the study may not be known with certainty. The potential for residual confounding also exists in our modeling results. The administrative databases may also lack information on some critical covariates such as PASI scores that could have accounted for psoriasis severity and been an important confounder for nonpersistence in this study. Third, health care claims are primarily used for payment and not research; hence, the presence of a pharmacy claim may not necessarily mean that the prescription was consumed as indicated. This could have had a negative impact on actual medication persistence. In addition, access to biologics may vary in individual commercial plans compared with those in the TRICARE program. Therefore, the persistence patterns may be partially reflective of the formulary access protocols. Finally, reasons for nonpersistence could not have been concluded with certainty due to the nature of the claims databases (lack of availability of critical variables mentioned above). Future research with a focus on real-world factors associated with nonpersistence among biologic users is needed, as is additional study on the impact of treatment compliance on overall and psoriasis-related costs for psoriasis-only and PsA-only subgroups.

\section{Conclusions}

Persistence on prevalent biologic treatment used for psoriasis and/or PsA was significantly associated with decreased total overall medical costs and psoriasis- and/or PsA-related total medical costs, primarily driven by lower ambulatory utilization costs. However, owing to the high unit costs and increased utilization of the biologics considered in the analysis, we observed significantly higher average overall and psoriasis- and/or PsArelated pharmacy costs in the persistent population. Total overall costs combining medical and pharmacy costs were similar between the persistent and nonpersistent patients. Future research will require better understanding of patient compliance to optimize treatment modalities for psoriasis.

\section{Authors}

SEINA LEE, MS, PharmD, Janssen Scientific Affairs, Lawrenceville, New Jersey. LIN XIE, MS, MA; YUEXI WANG, ME; and NEEL VAIDYA, MS, STATinMED Research, Ann Arbor, Michigan. ONUR BASER, MS, MA, PhD, STATinMED Research, Ann Arbor, Michigan, and Center for Innovation \& Outcomes Research, Department of Surgery, Columbia University, New York, New York.

AUTHOR CORRESPONDENCE: Yuexi Wang, ME, STATinMED Research, 211 N. Fourth Ave., Ste. 2B, Ann Arbor MI 48104. Tel.: 734.222.5426; E-mail: ywang@statinmed.com.

\section{DISCLOSURES}

This study was funded by Janssen Scientific Affairs. Lee is a paid employee of Janssen Scientific Affairs. Xie, Wang, Vaidya, and Baser are paid employees of STATinMED Research, which is a paid consultant to Janssen Scientific Affairs

This study was presented as an abstract at the Academy of Managed Care Pharmacy 2017 Annual Meeting, March 27-30, 2017, in Denver, CO.

\section{ACKNOWLEDGMENTS}

The authors thank Chris Haddlesey and Michael Moriarty of STATinMED Research for medical editorial support during manuscript development.

\section{REFERENCES}

1. Zhang XJ, Huang W, Yang S, et al. Psoriasis genome-wide association study identifies susceptibility variants within LCE gene cluster at lq21. Nat Genet. 2009;41(2):205-10.

2. Stern RS, Nijsten T, Feldman SR, Margolis DJ, Rolstad T. Psoriasis is common, carries a substantial burden even when not extensive, and is associated with widespread treatment dissatisfaction. J Investig Dermatol Symp Proc. 2004;9(2):136-39

3. Foster SA, Zhu B, Guo J, et al. Patient characteristics, health care resource utilization, and costs associated with treatment-regimen failure with biologics in the treatment of psoriasis. J Manag Care Spec Pharm. 2016;22(4):396-405. Available at: https://www.jmcp.org/doi/10.18553/jmcp.2016.22.4.396.

4. Brezinski EA, Dhillon JS, Armstrong AW. Economic burden of psoriasis in the United States: a systematic review. JAMA Dermatol. 2015;151(6):651-58.

5. Feldman SR, Zhao Y, Shi L, Tran MH. Economic and comorbidity burden among patients with moderate-to-severe psoriasis. J Manag Care Spec Pharm. 2015;21(10):874-88. Available at: https://www.jmcp.org/doi/10.18553/ jmcp.2015.21.10.874

6. Feldman SR, Zhao Y, Shi L, Tran MH, Lu J. Economic and comorbidity burden among moderate-to-severe psoriasis patients with comorbid psoriatic arthritis. Arthritis Care Res (Hoboken). 2015; 67(5):708-17.

7. Doshi JA, Takeshita J, Pinto L, et al. Biologic therapy adherence, discontinuation, switching, and restarting among patients with psoriasis in the U.S. Medicare population. J Am Acad Dermatol. 2016;74(6):1057-65.e4.

8. Anderson KL, Feldman SR. Reasons for treatment changes in patients with moderate to severe psoriasis. J Cutan Med Surg. 2015;19(4):361-66.

9. Harrold L, Stolshek B, Rebello S, et al. Impact of prior biologic use on persistence of treatment in patients with psoriatic arthritis enrolled in the U.S Corrona registry. Clin Rheumatol. 2017;36(4):895-901.

10. Zhang HF, Gauthier G, Hiscock R, Curtis JR. Treatment patterns in psoriatic arthritis patients newly initiated on oral nonbiologic or biologic disease-modifying antirheumatic drugs. Arthritis Res Ther. 2014;16(4):420. 


\section{Evaluating the Effect of Treatment Persistence on the Economic Burden of Moderate to Severe Psoriasis and/or Psoriatic Arthritis Patients in the U.S. Department of Defense Population}

11. Tian H, Gilloteau I, Mollon P, McDwyer P, Lotya J. Biologics switching patterns and associated costs in psoriasis patients in a large commercially insured population in the United States. Value Health. 2016;19(3):A125 [abstract].

12. Menter A, Augustin M, Signorovitch J, et al. The effect of adalimumab on reducing depression symptoms in patients with moderate to severe psoriasis: a randomized clinical trial. J Am Acad Dermatol. 2010;62:812-18.

13. Feldman SR, Evans C, Russell MW. Systemic treatment for moderate to severe psoriasis: estimates of failure rates and direct medical costs in a north-eastern U.S. managed care plan. J Dermatolog Treat. 2005;16(1):37-42.

14. Kimball AB, Guerin A, Tsaneva M, et al. Economic burden of comorbidities in patients with psoriasis is substantial. J Eur Acad Dermatol Venereol. 2011;25(2):157-63

15. Yu AP, Tang J, Xie J, et al. Economic burden of psoriasis compared to the general population and stratified by disease severity. Current Med Res Opin. 2009;25(10):2429-38.

16. Francis $\mathrm{K}, \mathrm{Yu} \mathrm{C}$, Alvrtsyan $\mathrm{H}$, et al. Healthcare utilization and costs associated with dabigatran compared to warfarin treatment in newly diagnosed patients with non-valvular atrial fibrillation. Curr Med Res Opin. 2015;31(12):2189-95.

17. Cheng J, Feldman SR. The cost of biologics for psoriasis is increasing Drugs Context. 2014;3:212266.

18. Patel V, Horn EJ, Lobosco SJ, Fox KM, Stevens SR, Lebwohl M. Psoriasis treatment patterns: results of a cross-sectional survey of dermatologists. J Am Acad Dermatol. 2008;58(6):964-69.

19. Grozdev I, Cao L, Kavlick K, Brodell RT, Mostow EN, Korman NJ. Psoriasis treatment patterns of dermatologists in northeast Ohio. J Cutan Med Surg. 2011;15(3):157-61.

20. Kimball AB, Gauthier G, Hiscock R, Zhang HF. Psoriasis treatment patterns: phototherapy, oral nonbiologic, and biologic therapies. Am J Pharm Benefits. 2015;7(2):e44-52.

21. Khalid JM, Fox KM, Globe G, Maguire A, Chau D. Treatment patterns and therapy effectiveness in psoriasis patients initiating biologic therapy in England. J Dermatolog Treat. 2014;25(1):67-72.
22. Kumar S, Kachhawha D, Das Koolwal G, et al. Psychiatric morbidity in psoriasis patients: a pilot study. Indian J Dermatol Venereol Leprol. 2011;77:625

23. Ferreira BI, Abreu JL, Dos Reis JP, Figueiredo AM. Psoriasis and associated psychiatric disorders: a systematic review on etiopathogenesis and clinical correlation. J Clin Aesthet Dermatol. 2016;9(6):36-43.

24. Dowlatshahi EA, Wakkee M, Herings RM, Hollestein LM, Nijsten T. Increased antidepressant drug exposure in psoriasis patients: a longitudinal population-based cohort study. Acta Derm Venereol. 2013;93(5):544-50.

25. Ogdie A, Harter L, Shin D, et al. The risk of fracture among patients with psoriatic arthritis and psoriasis: a population-based study. Ann Rheum Dis. 2017;76(5):882-85.

26. Wu CY, Chang YT, Juan CK, et al. Depression and insomnia in patients with psoriasis and psoriatic arthritis taking tumor necrosis factor antagonists. Medicine (Baltimore). 2016;95(22):e3816.

27. McDonough E, Ayearst R, Eder L, et al. Depression and anxiety in psoriatic disease: prevalence and associated factors. J Rheumatol. 2014;41(5):887-96.

28. Yélamos O, Ros S, Puig L. Improving patient outcomes in psoriasis: strategies to ensure treatment adherence. Psoriasis (Auckl). 2015;5:109-15.

29. Chapman BP, Moynihan J. The brain-skin connection: role of psychosocial factors and neuropeptides in psoriasis. Expert Rev Clin Immunol. 2009;5(6):623-27.

30. Levin AA, Gottlieb AB, Au SC. A comparison of psoriasis drug failure rates and reasons for discontinuation in biologics vs. conventional systemic therapies. J Drugs Dermatol. 2014;13(7):848-53

31. Foster SA, Zhu B, Guo J, et al. Patient characteristics, health care resource utilization, and costs associated with treatment-regimen failure with biologics in the treatment of psoriasis. J Manag Care Spec Pharm. 2016;22(4):396-405. Available at: https://www.jmcp.org/doi/10.18553/ jmcp.2016.22.4.396.

32. Sauer BC, Teng CC, He T, et al. Treatment patterns and annual biologic costs in U.S. veterans with rheumatic conditions or psoriasis. J Med Econ. 2016;19(1):34-43. 


\section{Evaluating the Effect of Treatment Persistence on the Economic Burden of Moderate to Severe Psoriasis and/or Psoriatic Arthritis Patients in the U.S. Department of Defense Population}

\section{APPENDIX A Treatments for Psoriasis and/or PsA}

Type

Treatment

CPT/HCPCS Codes if Applicable

Psoriasis- and/or PsA-related phototherapy

Photochemotherapy

Tar and ultraviolet B (Goeckerman treatment) or petrolatum and ultraviolet B PUVA

Goeckerman and/or PUVA for severe photoresponsive dermatoses requiring

24-8 hours of care under direct supervision of the physician (includes

application of medication and dressings)

Home phototherapy

Ultraviolet light therapy system panel, includes bulbs/lamps, timer, and eye protection

Photodynamic therapy

Application of light exposure followed by drug administration

Laser treatment for inflammatory

XTRAC Excimer Laser treatment

96910

96912

96913

skin disease

Nontopical systemic therapy for psoriasis and/or PsA

Biologics

\begin{tabular}{|l|l}
\hline Etanercept, injection & $\mathrm{J} 1438$ \\
\hline Adalimumab, injection & $\mathrm{J} 0135$ \\
\hline Ustekinumab, injection & $\mathrm{J} 3357$ \\
\hline Alefacept, injection & $\mathrm{J} 0215$ \\
\hline Efalizumab, injection & $\mathrm{S} 0162$ \\
\hline Apremilast, oral & \\
\hline Infliximab, injection & $\mathrm{J} 1745$ \\
\hline Acitretin & \\
\hline Isotretinoin & \\
\hline Cyclosporin & $\mathrm{J} 7502, \mathrm{~J} 7515, \mathrm{~J} 7516$ \\
\hline Methotrexate & $\mathrm{J} 8610, \mathrm{~J} 9250, \mathrm{~J} 9260$ \\
\hline Methoxsalen & \\
\hline Hydroxyurea & $\mathrm{S} 0176$ \\
\hline Thioguanine & \\
\hline Sulfasalazine & \\
\hline Tacrolimus & $\mathrm{J} 7503, \mathrm{~J} 7507, \mathrm{~J} 7508, \mathrm{~J} 7525$ \\
\hline Mycophenolate mofetil & $\mathrm{J} 7517$ \\
\hline Leflunomide & \\
\hline Azathioprine & $\mathrm{J} 7500, \mathrm{~J} 7501$ \\
\hline
\end{tabular}

Nonbiologics

E0691-E0694

96567, J7308

96920-96922

CPT = Current Procedural Terminology; HCPCS = Healthcare Common Procedure Coding System; PsA = psoriatic arthritis; PUVA =psoralen and ultraviolet A 


\section{Evaluating the Effect of Treatment Persistence on the Economic Burden of Moderate to Severe Psoriasis and/or Psoriatic Arthritis Patients in the U.S. Department of Defense Population}

\section{APPENDIX B Diagnosis Codes of Comorbid Conditions}

\begin{tabular}{|c|c|c|}
\hline Comorbid Conditions & ICD-9-CM Codes & ICD-10-CM Codes \\
\hline Diabetes mellitus & $250 . x x$ & E10.xx, El1.xx \\
\hline Inflammatory bowel disease & $555 . x, 556 . x$ & $\begin{array}{l}\text { K500.0, K500.10, K500.12, K500.13, K500.14, K500.18, K500.19, K501.0, } \\
\text { K501.11, K501.12, K501.13, K501.14, K501.18, K501.19, K508.0, K508.11, } \\
\text { K508.12, K508.13, K508.14, K508.18, K508.19, K509.0, K509.11, K509.12, } \\
\text { K509.14, K509.18, K509.19, K518.0, K512.0, K512.11, K512.12, K512.13, } \\
\text { K512.14, K512.18, K512.19, K513.0, K513.11, K513.12, K513.13, K513.14, } \\
\text { K513.18, K513.19, K514.0, K514.11, K514.12, K514.13, K514.14, K514.18, } \\
\text { K514.19, K515.0, K515.11, K515.12, K515.13, K515.14, K515.18, K515.19, } \\
\text { K510.0, K510.11, K510.12, K510.13, K510.14, K510.18, K510.19, K519.0, } \\
\text { K519.11, K519.12, K519.13, K519.14, K519.18, K519.19 }\end{array}$ \\
\hline Multiple sclerosis & 340 & G35 \\
\hline $\begin{array}{l}\text { Ankylosing spondylitis and axial } \\
\text { ankylosing spondylitis }\end{array}$ & 720 & M450-M459 \\
\hline Obesity & 278 & E66.0x, E66.1, E66.2, E66.8, E66.9, Z68.3xx, Z68.4xx \\
\hline Overweight & 278.02 & E66.3 \\
\hline Arteriosclerotic vascular disease & 429.2 & $\mathrm{I} 25.10$ \\
\hline Peripheral vascular disease & $\begin{array}{l}\text { 447.1, 557.1, 557.9, V43.4, 443.xx, } \\
093.0,437.3,440.0-441.9\end{array}$ & $\begin{array}{l}\text { I70.2xx, I70.3xx, I70.4xx, I70.5xx, I70.6xx, I70.7xx, I70.92, I73.9, I74.2-I72.4, } \\
\text { I75.0xx }\end{array}$ \\
\hline Cerebrovascular ischemia & $435.9,437.1$ & G45.9, I67.841, I67.848 \\
\hline Atherosclerosis & $440 . x x$ & $\begin{array}{l}\text { I70.0, I70.1, I70.201-I70.203, I70.208, I70.209, I70.90, I70.91, I70.211-I70.213, } \\
\text { I70.218, I70.219, I70.221-I70.223, I70.228, I70.229, I70.231-I70.235, } \\
\text { I70.238, I70.239, I70.241-I70.245, I70.248, I70.249, I70.25, I70.261-I70.263, } \\
\text { I70.268, I70.269, I70.291-I70.293, I70.298, I70.299, I70.301-I70.303, } \\
\text { I70.308, I70.309, I70.311-170.313, I70.318, I70.319, I70.321-I70.323, I70.328, } \\
\text { I70.329, I70.331, I70.401-I70.403, I70.408, I70.409, I70.411-I70.413, I70.418, } \\
\text { I70.419, I70.421-I70.423, I70.428, I70.429, I70.431-I70.435, I70.438, I70.439, } \\
\text { I70.441-I70.445, I70.448, I70.449, I70.461-I70.463, I70.468, I70.469, } \\
\text { I70.491-I70.493, I70.498, I70.499, I70.501-I70.503, I70.508, I70.509, } \\
\text { I70.511-I70.513, I70.518, I70.519, I70.521-I70.523, I70.528, I70.529, } \\
\text { I70.531-I70.535, I70.538, I70.539, I70.541-I70.545, I70.548, I70.549, I70.50, } \\
\text { I70.561-I70.563, I70.568, I70.569, I70.591-I70.593, I70.598, I70.599. }\end{array}$ \\
\hline Dyslipidemia & 272.xx & $\begin{array}{l}\text { E78.0-E78.7, E78.81, E78.89, E78.9, E75.21, E75.22, E75.249, E75.6, E77.0, } \\
\text { E77.1, E88.89 }\end{array}$ \\
\hline Hypertension & $\begin{array}{l}\text { 401.x, 402.xx, 403.xx, 404.xx, } \\
\text { 405.xx, 437.2 }\end{array}$ & I10-I15.x, I67.4 \\
\hline Stroke & $434 . x x$ & $\begin{array}{l}\text { I66.09, I66.19, I66.29, I66.9, I66.30, I66.311, I66.312, I66.319, I66.321, I66.322, } \\
\text { I66.329, I66.331, I66.332, I66.339, I66.341, I66.342, I66.349, I66.39, I66.40, } \\
\text { I66.411, I66.412, I66.419, I66.421, I66.422, I66.429, I66.431, I66.432, I66.439, } \\
\text { I66.441, I66.442, I66.449, I66.49, I66.50, I66.511, I66.512, I66.519, I66.521, } \\
\text { I66.522, I66.529, I66.531, I66.532, I66.539, I66.541, I66.542, I66.549, I66.59, } \\
\text { I66.01-I66.03, I66.11-I66.13, I66.19, I66.21-I66.23, I66.29, I66.3, I66.8, I63.8, } \\
\text { I63.9, I63.50, I63.511, I63.512, I63.519, I63.521, I63.522, I63.529, I63.531, } \\
\text { I63.532, I63.539, I63.541, I63.542, I63.549 }\end{array}$ \\
\hline Myocardial infarction & $410.0-410.92,412 . x x$ & I21.xx, I22.x, I25.2 \\
\hline Coronary artery disease & $414 . x x$ & $\begin{array}{l}\text { I25.701, I25.709, I25.711, I25.718, I25.719, I25.721, I25.728, I25.729, I25.731, } \\
\text { I25.738, I25.119, I25.739, I25.751, I25.758, I25.759, I25.761, I25.768, I25.769, } \\
\mathrm{I} 25.791, \mathrm{I} 25.798, \mathrm{I} 25.799, \mathrm{I} 25.750, \mathrm{I} 25.751, \mathrm{I} 25.758, \mathrm{I} 25.811, \mathrm{I} 25.760, \mathrm{I} 25.761, \\
\mathrm{I} 25.768, \mathrm{I} 25.769, \mathrm{I} 25.810-\mathrm{I} 25.812, \mathrm{I} 25.82-\mathrm{I} 25.84, \mathrm{I} 25.41, \mathrm{I} 25.42, \mathrm{I} 25.10, \mathrm{I} 25.3, \\
\mathrm{I} 25.5, \mathrm{I} 25.6, \mathrm{I} 25.89, \mathrm{I} 25.9\end{array}$ \\
\hline
\end{tabular}

